\title{
Efeitos do Processo de Refusão a Laser na Microestrutura e No Comportamento de Desgaste do Revestimento de WC Aspergido com HVOF
}

\section{Effects of Laser Remeltina Process on \\ Microstructure and Wear Behavior of WC Coating Sprayed by HVOF}

Renato Camponogara Panziera ${ }^{1}$, Ana Claudia Costa de Oliveira ${ }^{2}$

\footnotetext{
${ }^{1}$ Engenheiro Mecânico, Universidade Federal do Pampa - UNIPAMPA, CEP: 97546-550, Alegrete, RS, Brasil.

${ }^{2}$ Departamento de Engenheira Mecânica, Universidade Federal do Pampa - UNIPAMPA, CEP: 97546-550, Alegrete, RS, Brasil.

e-mail: renato.panziera@ hotmail.com, aclaudiacosta21@gmail.com.
}

\begin{abstract}
RESUMO
O desgaste de componentes e equipamentos industriais e agrícolas representa um grande fator de depreciação de capital e de fonte de despesas com manutenção e reposição de componentes mecânicos. Como o desgaste é um fenômeno essencialmente superficial, envolvendo a remoção mecânica indesejável de material de superfícies, a solução estudada nesse trabalho foi analisar o comportamento do desgaste abrasivo de uma superfície revestida com Carbeto de Tungstênio (WC) aspergido pelo método de aspersão térmica oxi-combustível de alta velocidade, conhecida como HVOF (do inglês High Velocity Oxygen Fuel), sobre um substrato de aço SAE 1020. Para melhorar a adesão entre o revestimento e substrato, diminuir a porosidade presente no revestimento e obter melhores resultados no ensaio de desgaste abrasivo foi realizado um tratamento com laser de $\mathrm{CO}_{2}$, cuja potência utilizada foi de $125 \mathrm{~W}$, na superfície do revestimento de WC. As amostras revestidas via $\mathrm{HVOF}$ e $\mathrm{HVOF}$ + tratamento com laser de $\mathrm{CO}_{2}$ foram caracterizadas através de Microscopia Eletrônica de Varredura (MEV) para avaliar a morfologia e microestrutura do revestimento e interface, análise de microdureza, ensaio de desgaste e medida da rugosidade foram realizados. Obteve-se como resultado que a amostra revestida pelo método HVOF apresentou um melhor desempenho em ensaios de microdureza, desgaste e rugosidade em comparação com a amostra tratada com o laser.
\end{abstract}

Palavras-chave: aspersão térmica, desgaste, HVOF, laser.

\begin{abstract}
The wear of industrial and agricultural components and equipment represents a major factor of depreciation of capital and source of expenses with maintenance and replacement of mechanical components. As the wear is an essentially superficial phenomenon, involving the undesirable mechanical removal of surface materials, the solution studied in this work was to analyze the abrasive wear behavior of a surface coated with Tungsten Carbide (WC) sprayed by the thermal spray method by High Velocity Oxygen Fuel - HVOF on a SAE 1020 steel substrate. To improve the adhesion between the coating and substrate, to reduce the porosity present in the coating and to obtain better results in the abrasive wear test, a $\mathrm{CO}_{2}$ laser treatment with a power of $125 \mathrm{~W}$ was applied to the surface of the WC coating. The samples coated by $\mathrm{HVOF}$ and $\mathrm{HVOF}+\mathrm{CO}_{2}$ laser treatment were characterized by Scanning Electron Microscopy (SEM) to evaluate the morphology and microstructure of the coating and interface, microhardness analysis, wear and roughness test were done. It was obtained as a result that the sample coated by the HVOF method showed a better performance in tests of microhardness, wear and roughness compared to the sample treated with the laser.
\end{abstract}

Keywords: thermal spray, wear, HVOF, laser.

\section{INTRODUÇÃO}

Atualmente, a diversidade de opções para o uso de materiais, para obter um melhor desempenho e menor custo, fez com que o ramo da engenharia de superfície tenha crescido consideravelmente nas últimas décadas. O desgaste é um dos principais problemas encontrados em peças e equipamentos de máquinas agrícolas que 
trabalham em culturas de cereais. Isto é devido ao fato de que o grão geralmente apresenta uma textura que o torna abrasivo, como no caso do grão de arroz com casca. Desta forma, o equipamento que tenha contato direto com o grão terá uma vida útil menor do que as outras partes [1].

Os métodos por aspersão térmica visam a necessidade de aumentar a vida útil de componentes e peças, permitindo a reutilização dessas com deposição de materiais metálicos ou não metálicos sobre os componentes devidamente preparados [2]. Neste processo, existem várias variáveis envolvidas que, ao atuar em conjunto e aplicadas corretamente, produzem um resultado melhor do que se considerado individualmente. $\mathrm{O}$ material de revestimento pode ser na forma de pó, vareta, cordão ou arame [3].

Para o processo Oxi-combustível de Alta Velocidade (HVOF - High Velocity Oxygen Fuel), o material de revestimento está na forma de pó. Por meio deste método, o pó que servirá de revestimento no substrato é expelido por meio de uma tocha de aspersão específica que opera sob alta pressão. Através da combustão de um combustível e oxigênio, as partículas do pó são fundidas ou parcialmente fundidas e jogadas com velocidades de até $1200 \mathrm{~m} / \mathrm{s}$ contra o substrato de acordo com LIMA e TREVISAN [4].

De acordo com CASTRO et al. [5], a aplicação de revestimento de carboneto de tungstênio através de aspersão térmica aumentará a durabilidade e reduzirá o desgaste abrasivo, de modo que este procedimento pode ser aplicado a equipamentos e peças de máquinas agrícolas que tenham contato com o grão de arroz com casca. De acordo com GARCIA-ALONSO et al. [6] em seu estudo sobre a utilização de laser sobre revestimentos, o tratamento a laser do revestimento aspergido foi considerado um meio eficiente para densificar esse revestimento, conseguindo o refinamento da estrutura e o aprimoramento da adesão do revestimento/substrato. Isto é devido à homogeneização e eliminação de poros e lamelas do revestimento aspergido. Além disso, acredita-se que a ligação metalúrgica seja estabelecida após a refusão do revestimento conforme SIDHU et al. [7] e TELEGINSKI et al. [8].

A fusão a laser é uma técnica bem estabelecida, muitas vezes usada para reduzir defeitos inerentes, como a porosidade dos revestimentos em pó. Além disso, a técnica de laser surface alloying (LSA) é uma área de pesquisa relativamente nova e importante, que também é usada para melhorar as propriedades resistentes ao desgaste das superfícies. O objetivo dessas técnicas é eliminar completamente os poros dentro dos revestimentos para que o processo de fusão e solidificação resulte em um revestimento homogêneo [9-11].

De acordo com GANEEV [12], o uso de um laser de $\mathrm{CO}_{2}$ com parâmetros de potência de $100 \mathrm{~W}$, diâmetro do feixe de $0,1 \mathrm{~mm}$, duração do pulso de $6 \mathrm{~ns}$, repetição da frequência de pulso $20 \mathrm{~Hz}$ e comprimento da onda de $1064 \mathrm{~nm}$ foi suficiente para produzir padrões de extinção superficial em aço carbono anteriormente revestido com grafite cuja dureza resultante do tratamento a laser foi de $950 \mathrm{HV}$, com uma profundidade de $170 \mu \mathrm{m}$. No estudo de CONTIN et al. [13], a aplicação da formação de barreiras intermediárias, feita por processo a laser foi eficiente para aumentar a ligação do revestimento de carbono superficial.

Conforme LIMA e TREVISAN [4], dentre os vários métodos de aspersão térmica, o processo via HVOF é um dos que apresenta melhor resistência adesiva, elevada densidade e dureza quando utilizado pó de WC como revestimento.

Dentro desse contexto, este estudo tem por objetivo explorar o uso da deposição de Carbeto de Tungstênio (WC) via HVOF e o processo de refusão a laser, a fim de obter melhores propriedades contra o desgaste abrasivo de componentes de máquinas agrícolas em contato com a casca de grão de arroz, já que esse é um dos principais problemas enfrentados pelos orizicultores.

\section{MATERIAIS E MÉTODOS}

O material do substrato é o aço SAE 1020 com 0,20\% de carbono. As amostras são retangulares com dimensões de $60 \mathrm{~mm}$ X $22 \mathrm{~mm}$ X $10 \mathrm{~mm}$. A preparação da superfície dos corpos de prova consistiu simplesmente em jateamento abrasivo por granalha de $\mathrm{Al}_{2} \mathrm{O}_{3}$ de granulação de 120 mesh para promover a rugosidade da superfície, para uma melhor aderência do revestimento. Para a aplicação do revestimento de carboneto de tungstênio por aspersão térmica HVOF, foi utilizado um aparelho de aspersão Praxair-TAFA JP-5000 HP/HVOF controlado por um braço robótico Praxair Model 1270.

O pó utilizado no processo de aspersão térmica foi o WOKA 3653 (WC-10Co-4Cr), fornecido pela empresa Harris Brastak Soldas especiais S.A. A composição química do pó de Carbeto de Tungstênio esta apresentado na Tabela 1 conforme a norma standard METCO. 
Tabela 1: Composição química do pó utilizado no processo de aspersão térmica.

\begin{tabular}{|c|c|c|c|}
\hline ELEMENTO & $\begin{array}{c}\text { ESPECIFICADO [\% } \\
\text { EM PESO] }\end{array}$ & $\begin{array}{c}\text { ENCONTRADO [\% } \\
\text { EM PESO] }\end{array}$ & $\begin{array}{c}\text { DENSIDADE } \\
\text { [g/cm } \mathbf{c m}^{\mathbf{3}}\end{array}$ \\
\hline $\mathrm{C}$ & $4,500 / 7,500$ & 5,320 & \multirow{2}{*}{5} \\
\cline { 1 - 3 } $\mathrm{Co}$ & $9,000 / 11,000$ & 10,380 & \multirow{2}{*}{5,0} \\
\cline { 1 - 3 } $\mathrm{Cr}$ & $3,000 / 4,500$ & 4,230 & \\
\hline $\mathrm{W}$ & $\begin{array}{c}\text { RESTANTE / RES- } \\
\text { TANTE }\end{array}$ & 80,070 & \\
\hline
\end{tabular}

Após a aspersão do pó de carboneto de tungstênio (WC), as amostras foram irradiadas por um feixe de laser de $\mathrm{CO}_{2}$, com potência de $125 \mathrm{~W}$, comprimento de onda de $10,6 \mu \mathrm{m}$ e distribuição de energia Gaussiana, marca SYNRAD, modelo Evolution 125, com o objetivo de melhorar a adesão entre o revestimento e substrato, diminuir a porosidade presente no revestimento e obter melhores resultados nos ensaios. Inicialmente, foram analisados 4 parâmetros diferentes nas condições de laser. O parâmetro 1 (P1) foi escolhido como a melhor condição obtida, que será discutido posteriormente, para realização das análises. O feixe de laser foi direcionado para a superfície das amostras por espelhos galvanométricos localizados nas cabeças de varredura FH Flyer Marking Head.

A Tabela 2 mostra a carta de processo para o tratamento da superfície com feixe de laser, variando a duração do pulso do laser e taxa de sobreposição (resolução).

Tabela 2: Carta de processos de Laser de $\mathrm{CO}_{2}$ com $100 \%$ de potência (125W).

\begin{tabular}{|c|c|c|c|}
\hline Parâmetros & Número de passes do feixe de laser & Duração do pulso (ms) & Resolução \\
\hline \multirow{3}{*}{ P1 } & $1^{\mathrm{a}}$ & 10 & 300 \\
\cline { 2 - 4 } & $2^{\mathrm{a}}$ & 25 & 127 \\
\hline \multirow{3}{*}{ P2 } & $1^{\mathrm{a}}$ & 10 & 300 \\
\cline { 2 - 4 } & $2^{\mathrm{a}}$ & 10 & 300 \\
\hline \multirow{3}{*}{ P3 } & $1^{\mathrm{a}}$ & 10 & 300 \\
\cline { 2 - 4 } & $2^{\mathrm{a}}$ & 25 & 127 \\
\cline { 2 - 4 } & $3^{\mathrm{a}}$ & 10 & 300 \\
\hline \multirow{3}{*}{ P4 } & $1^{\mathrm{a}}$ & 10 & 300 \\
\cline { 2 - 4 } & $2^{\mathrm{a}}$ & 10 & 300 \\
\cline { 2 - 4 } & $3^{\mathrm{a}}$ & 10 & 300 \\
\hline
\end{tabular}

A amostra revestida e irradiada com o feixe de laser juntamente com os 4 parâmetros da carta de processos é apresentada na Figura 1.

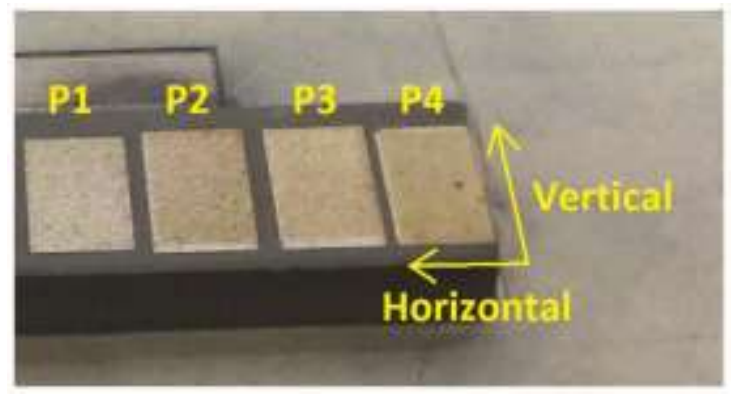

Figura 1: Foto do corpo de prova com os 4 parâmetros irradiados com laser.

\subsection{Caracterização do material}

Para a análise microestrutural de alta resolução e determinação da espessura do revestimento das amostras 
revestidas com WC e posteriormente irradiadas com laser de $\mathrm{CO}_{2}$, utilizou-se um microscópio eletrônica de varredura (MEV) modelo EVO MA10 da marca Zeiss Mark.

Para medidas de microdureza, um microdurômetro da marca Buehler, modelo MicroMet 6010 foi usado. Uma carga de $100 \mathrm{~g}$ foi utilizada para realizar as indentações, tanto no revestimento como no substrato, para assim, ser possível mensurar a microdureza ao longo do revestimento e substrato.

Para realização do ensaio de desgaste, utilizou-se o equipamento Phoenix Tribology, modelo TE 53 SLIM, cujo princípio de funcionamento é o de bloco sobre anel. Após ensaios preliminares, com cargas aplicadas de $1 \mathrm{~kg}, 2 \mathrm{~kg}, 3 \mathrm{~kg}$ e $5 \mathrm{~kg}$, lixas d'água de granulometria de 50, 80, 120 e 150 mesh e com 100, 200, $300,400,500$ e 650 revoluções, foi possível definir um parâmetro operacional da máquina para obter um desgaste.

Os parâmetros utilizados para realizar o ensaio de desgaste foram:

- Diâmetro do disco de desgaste de $60 \mathrm{~mm}$;

- Velocidade de $50 \mathrm{rpm}(0,15 \mathrm{~m} / \mathrm{s})$;

- Massa aplicada de $5 \mathrm{kgf}$ convertida em um carga de $287 \mathrm{~N}$ pela máquina;

- Lixa d'água de $120 \mathrm{mesh} / \mathrm{cm}^{2}$ (colada sobre o disco de desgaste);

- Número de ciclos de 650.

Para medir a perda de massa no ensaio de desgaste, utilizou-se uma balança de precisão com resolução de $0,001 \mathrm{~g}$ da marca Shimadzu modelo AY220, cujo objetivo é medir a massa das amostras limpas antes e após o ensaio e, através desta diferença, calcular a perda de massa (Perda de massa $\left.=m_{i}-m_{0}\right)$, onde $m_{i}$ é a massa inicial e $\mathrm{m}_{0}$ é a massa final da amostra. A perda de volume das amostras foi obtida através da Equação 1.

$$
\text { Perda de volume }=\frac{\text { perda de massa }}{\text { densidade }(\rho)} \times 1000\left[\mathrm{~mm}^{3}\right]
$$

Onde: Perda de massa $=[\mathrm{g}]$ e densidade $=\left[\mathrm{g} / \mathrm{cm}^{3}\right]$. lo TR200.

Para a medida da rugosidade média, utilizou-se o rugosímetro digital portátil da marca HOMIS, mode-

\section{RESULTADOS E DISCUSSÃO}

As partículas de WC com morfologia arredondada e tamanho médio de grão $39,2 \mu \mathrm{m}$ são mostradas na Fig. 2. De acordo com um estudo de VAN ACKER et al. [14], o tamanho das partículas de pó pode influenciar o comportamento de desgaste dos revestimentos aspergidos. O tamanho médio de grão encontrado através da análise de MEV para o presente estudo é de $39 \pm 5 \mu \mathrm{m}$, o que está dentro da faixa recomendada para esse processo. A morfologia esférica do pó proporciona um bom fluxo das partículas na pistola de aspersão [15].

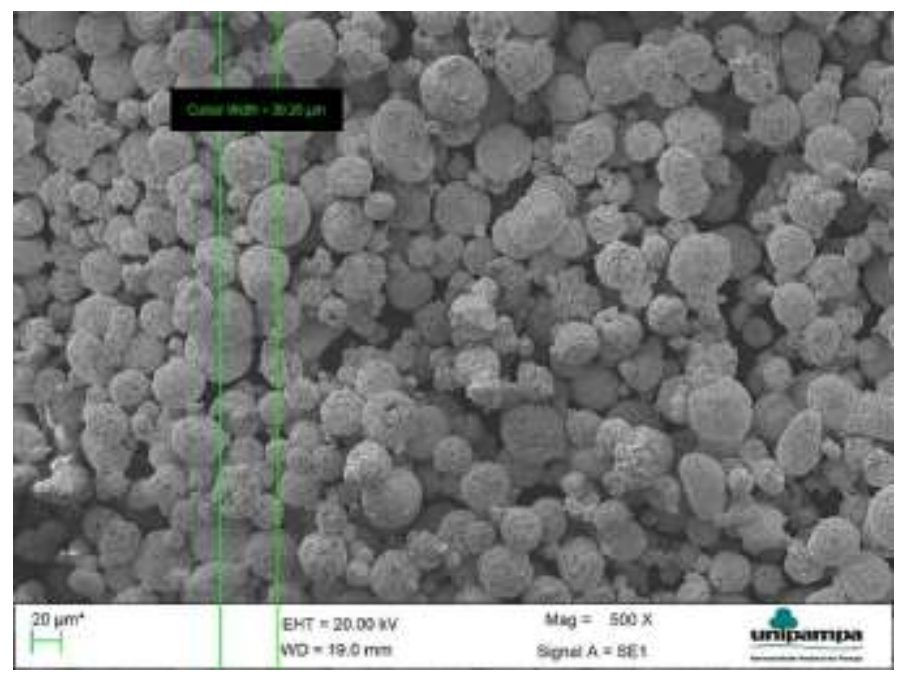

Figura 2: Imagem do pó de WC.

A análise de MEV na seção de interface foi utilizada para determinar a espessura do revestimento de 
WC aspergido pelo método HVOF e a aderência, como pode ser visto na Figura 3. Foi encontrada uma espessura média de 325,2 $\mu \mathrm{m}$ e visualmente o revestimento apresenta um bom ancoramento mecânico com o substrato, poucos poros, óxidos e partículas não fundidas de WC.

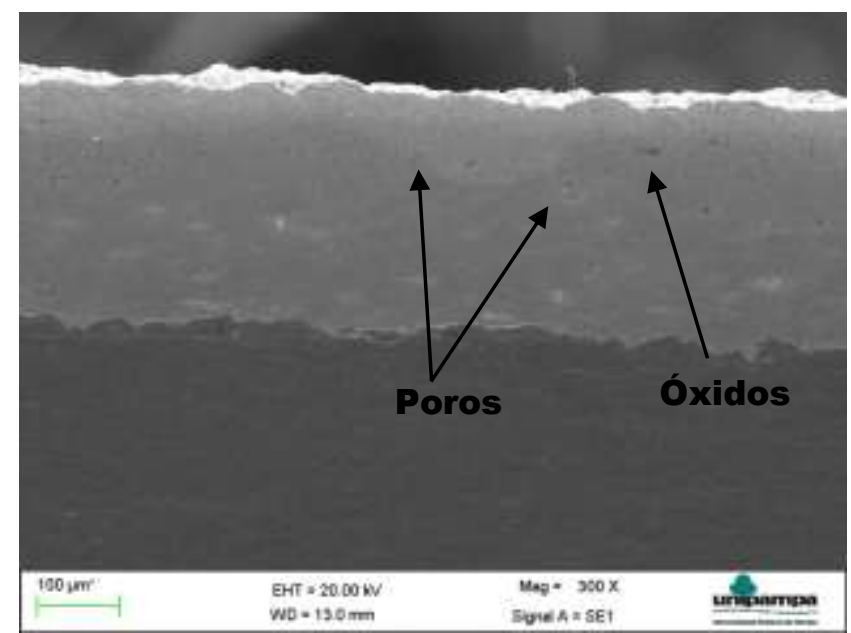

Figura 3: Espessura do revestimento por MEV.

A Figura 4 mostra a análise do revestimento com o tratamento a laser de $\mathrm{CO}_{2}$. Pode ser visto que na Figura 4 (b), (c) e (d) o revestimento apresenta trincas superficiais e poros mais próximos do substrato apresentando também uma má fusão do laser nessa região. A Figura 4 (a) mostra que o parâmetro utilizado foi capaz de diminuir a porosidade do revestimento deixando o revestimento mais homogêneo, mas ainda não foi o suficiente para ocorrer uma ligação metalúrgica.

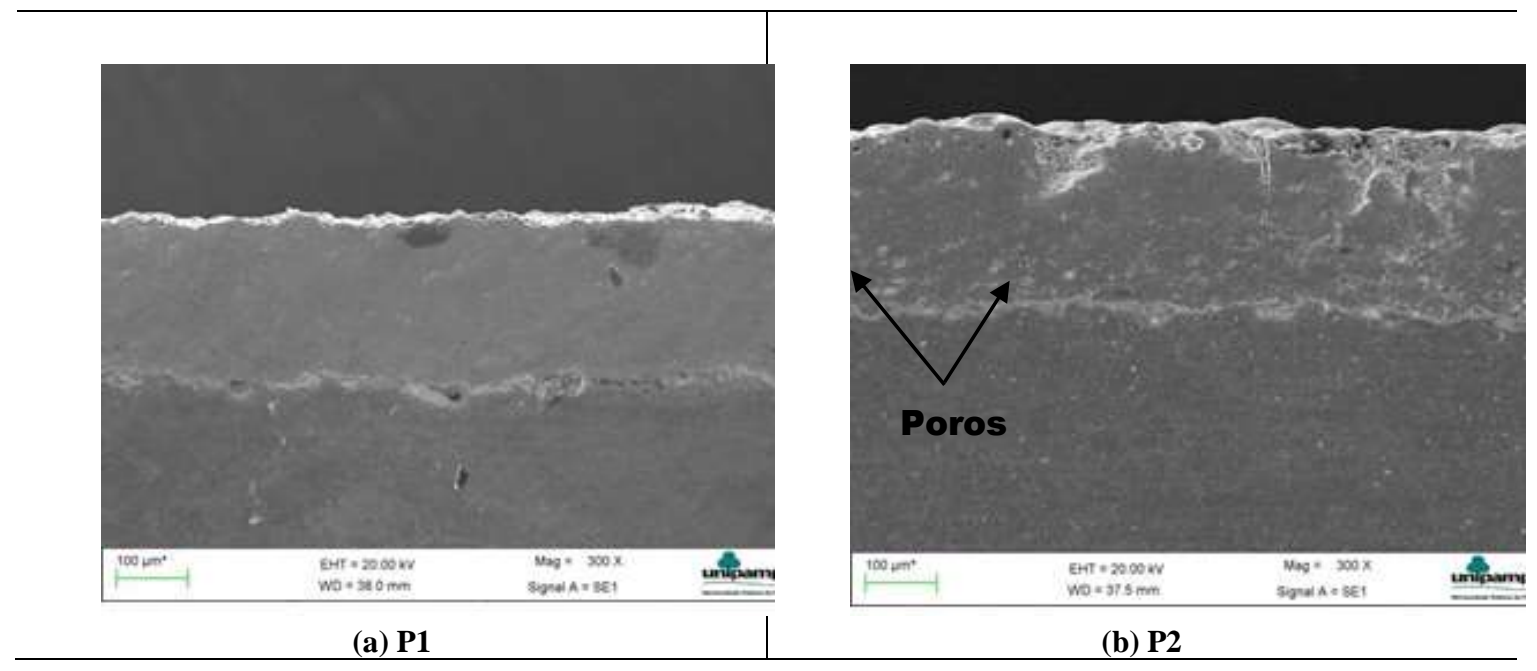




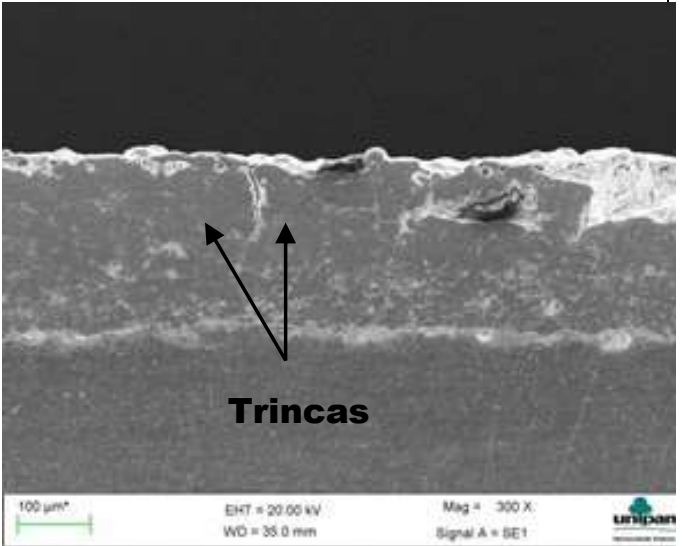

(c) P3

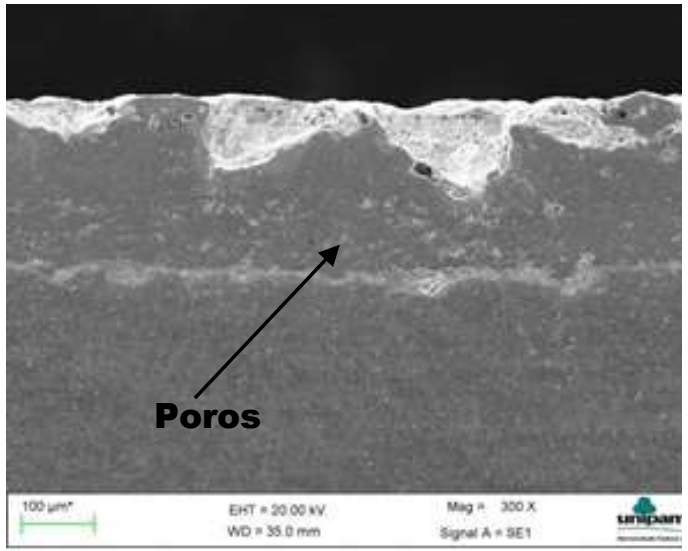

(d) $\mathbf{P 4}$

Figura 4: Imagens do revestimento de WC com tratamento a Laser de $\mathrm{CO}_{2}$ para os Parâmetros de 1 a 4.

No entanto, o fato da refusão não atingir a extensão total do revestimento indica que o tempo de interação entre o laser e o material não foi suficiente para promover um aumento de temperatura até atingir o fusão do revestimento em toda a sua extensão. De acordo com MATEOS et al. [16], em muitas ocasiões, observa-se que a refusão parcial das camadas causam seu desprendimento da base metálica.

Após a análise no MEV, o P1 foi escolhido para realização dos ensaios de desgaste, microdureza e rugosidade por apresentar pouca porosidade.

\subsection{Microdureza}

O ensaio de microdureza foi realizado na escala Vickers (HV) com carregamento de carga de 0,1 kg. A Figura 5 apresenta os resultados das medições de microdureza na amostra revestida com WC e amostra com tratamento de laser P1.

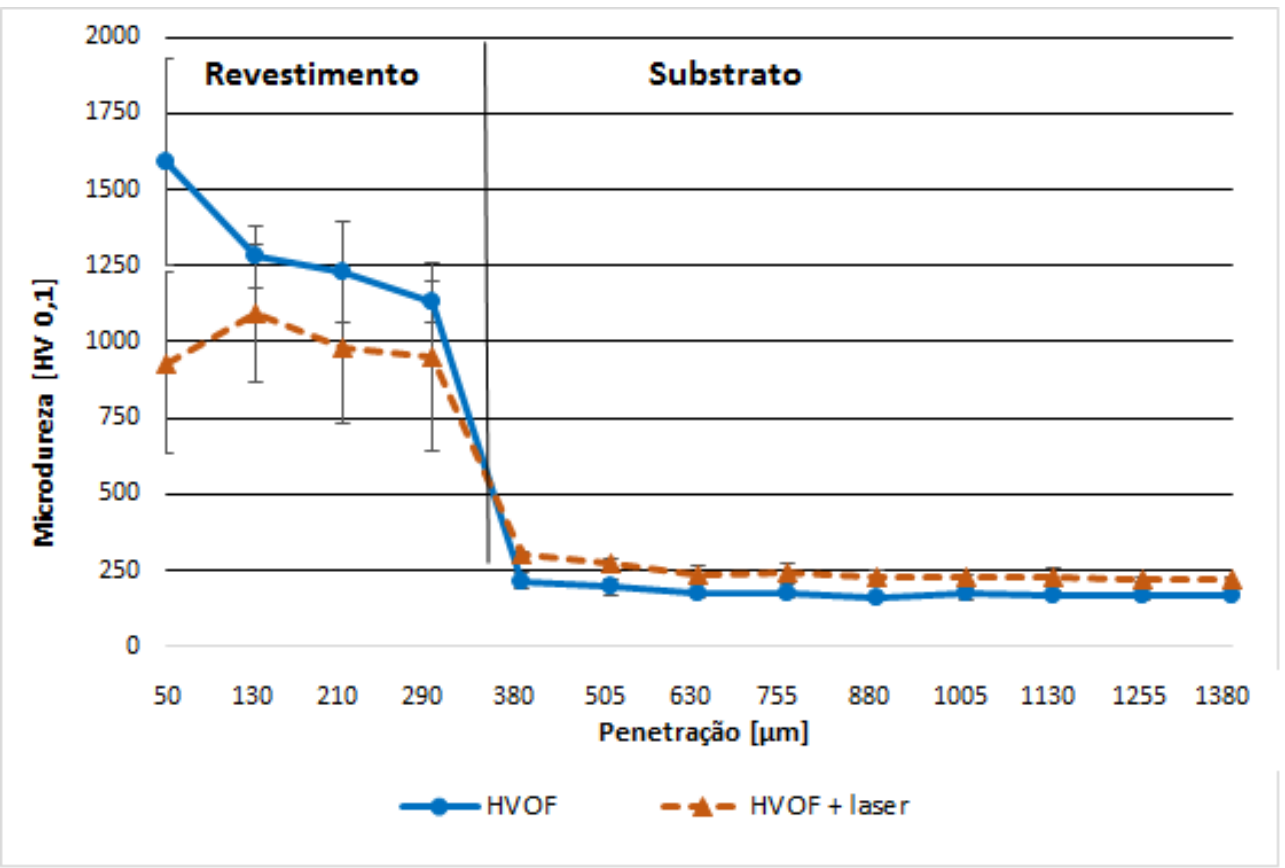

Figura 5: Efeito da microdureza com revestimento de WC via HVOF e após tratamento com laser de $\mathrm{CO}_{2}$.

Pode-se perceber que a amostra revestida com WC sem a irradiação do laser, linha contínua, apresentou uma microdureza superior, obtendo uma média de dureza próxima a $1560 \mathrm{HV}$ com desvio padrão amos- 
tral de 337 para a primeira indentação. Na segunda, terceira e quarta indentação, ainda sobre o revestimento, a média ficou entre $1278 \mathrm{HV}$ e $1131 \mathrm{HV}$ com desvio padrão menor entre 67 e 165. Para as nove restantes indentações sobre o substrato de aço 1020 a microdureza se manteve abaixo dos $250 \mathrm{HV}$. Espera-se que os valores de alta dureza das amostras revestidas na aquisição de amostras não revestidas sejam maiores mesmo em condições sem tratamento a laser, uma vez que as partículas de WC apresentam alta dureza, 3000$4000 \mathrm{HV}$ e são estáveis a altas temperaturas. Esta propriedade torna-o atraente para uso em revestimentos resistentes ao desgaste [14].

Por fim, a amostra revestida com WC irradiada com Laser de $\mathrm{CO}_{2}$, Figura 5 com linha pontilhada, cujo parâmetro escolhido foi o P1 da carta de processos, mostrou que os valores de microdureza sobre o revestimento mantiveram-se em torno de 1000 HV. Diferentemente do caso anterior, a segunda indentação passa a apresentar uma microdureza relativamente superior as demais sobre o revestimento, podendo ser caracterizada pelo processo de resfriamento da refusão do laser. As demais indentações também mantiveram-se abaixo de $250 \mathrm{HV}$, salvo as duas primeiras indentações sobre o substrato que podem ter sofrido os efeitos do laser por terem apresentado uma microdureza superior aos $250 \mathrm{HV}$.

\subsection{Ensaio de desgaste}

Os resultados obtidos no ensaio de desgaste por perda de volume realizado com lixa d'água de $120 \mathrm{mesh} / \mathrm{pol}^{2}$ colada sobre o disco de desgaste, 650 ciclos, velocidade de $50 \mathrm{rpm}$ e carga aplicada de $5 \mathrm{kgf}$ são apresentados na Figura 6. O aço revestido com WC sem tratamento a laser e outro corpo de prova irradiado são analisados juntamente com a amostra de aço.

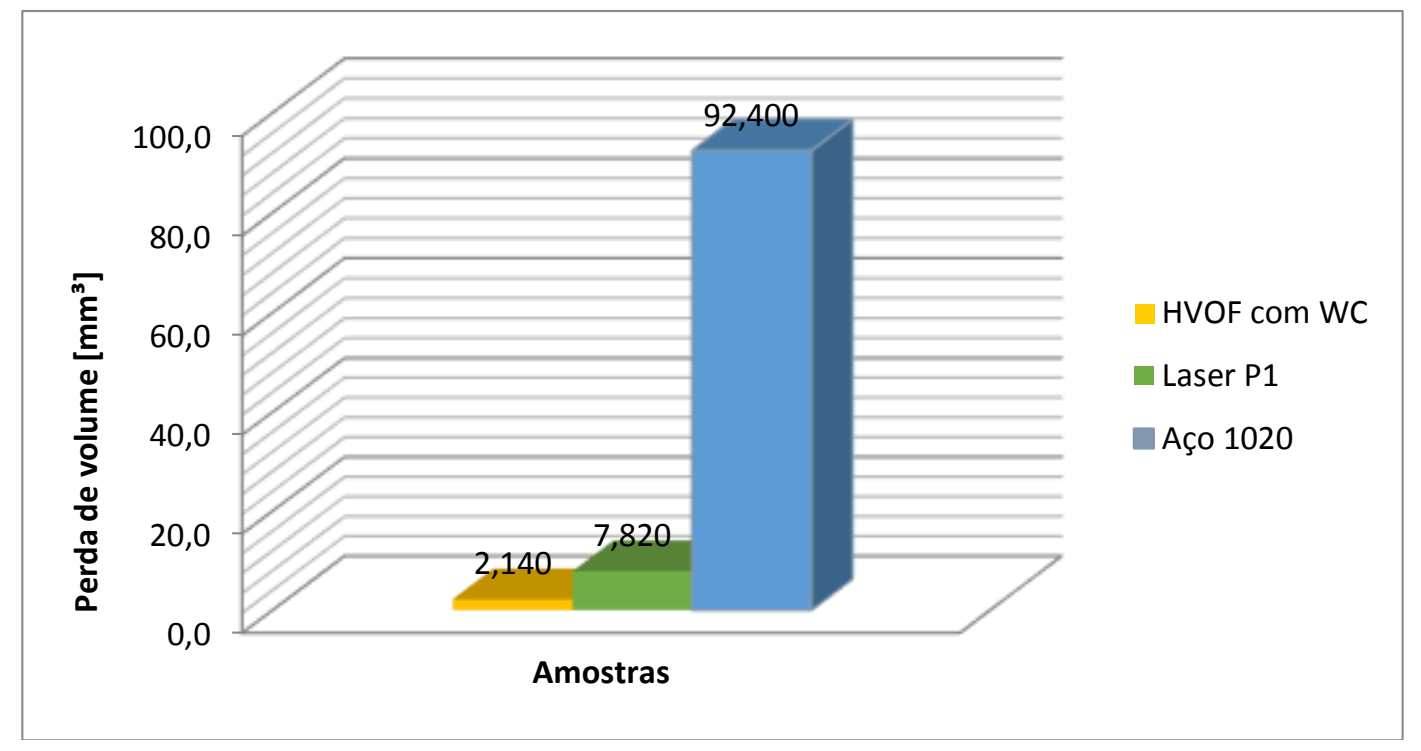

Figura 6: Resultados do ensaio de desgaste por perda de volume.

Pode-se notar que o aço 1020 sem revestimento teve a maior perda de volume em relação às demais amostras testadas e a amostra aspergida pelo método HVOF e revestida com pó de WC teve a menor perda de volume. É possível notar que a diferença da perda de volume da amostra de aço 1020 foi: 11,8 vezes maior do que a amostra revestida com carboneto de tungstênio e irradiada com laser pulsado $\mathrm{P} 1$ de $\mathrm{CO}_{2} ; 43,17$ vezes maior do que a amostra revestida com carboneto de tungstênio sem a irradiação a laser.

Comparando apenas a amostra revestida com carboneto de tungstênio e revestida com WC e com irradiação a laser, é possível perceber que houve uma perda de volume de 3,65 vezes menor na amostra sem irradiação a laser. Portanto, é possível reconhecer que ambos são mais resistentes ao desgaste abrasivo em comparação com a outra amostra. No entanto, a diminuição da microdureza na amostra irradiada por laser pode ter sido o fator determinante para o aumento da perda de volume neste ensaio. No estudo realizado por ZHANG et al. [11], maiores valores de dureza após o tratamento a laser em revestimentos de HVOF são acompanhados por melhora nos ensaios de desgaste.

O desempenho dos revestimentos com tratamento com laser é provavelmente devido aos parâmetros do processo P1 empregados. De acordo com MATEOS et al. [16] os parâmetros da aplicação laser podem 
causar rachaduras nos revestimentos, bem como a manutenção de valores de porosidade para valores elevados que podem comprometer seu desempenho. Neste estudo, os valores de alta energia utilizados durante o tratamento levaram à produção de revestimentos de baixa qualidade. No entanto, o uso de menor intensidade de energia contribuiu para a melhoria das propriedades dos revestimentos.

\subsection{Rugosidade}

A Tabela 3 mostra a medida da rugosidade média para amostras tratadas com HVOF e Laser Pulsado. O ensaio feito com a amostra com laser pulsado foi realizado com o P1. É importante enfatizar a importância de ter uma peça com baixa rugosidade, uma vez que as colheitadeiras de arroz devem ter em seus equipamentos uma superfície lisa, para que o grão de arroz deslize e não venha travar o fluxo de arroz dentro do equipamento, além de não ter o grave problema de descascar o grão.

Tabela 3: Resultados das medidas de rugosidade.

\begin{tabular}{|c|c|c|}
\hline \multicolumn{3}{|c|}{ RUGOSIDADE } \\
\hline & HVOF & LASER \\
\hline Medições & Ra $[\boldsymbol{\mu m}]$ & Ra $[\boldsymbol{\mu m}]$ \\
\left.\hline${\text { Este } 1^{\text {a }}}^{\mathbf{a}}\right]$ & 5233 & 5736 \\
\hline $2^{\text {a }}$ & 4415 & 6190 \\
\hline $3^{\text {a }}$ & 4114 & 6425 \\
\hline $4^{\text {a }}$ & 3786 & 5941 \\
\hline $5^{\text {a }}$ & 4831 & 5825 \\
\hline Desvio Padrão & 572,26 & 281,86 \\
\hline Média & $\mathbf{4 4 7 5 , 8 0}$ & $\mathbf{6 0 2 3 , 4 0}$ \\
\hline
\end{tabular}

Pode-se perceber que a amostra apenas revestida com WC apresentou uma rugosidade média Ra menor do que a amostra irradiada com laser pulsado. Vale ressaltar que este problema pode ser corrigido com um polimento superficial do revestimento caso essa rugosidade faça com que os grãos de arroz venham a descascar durante o processo de colheita, no entanto, esse artifício levaria a um custo maior.

\section{CONCLUSÕES}

A aplicação do revestimento de carboneto de tungstênio via HVOF apresentou baixa porosidade e nenhuma trinca. Em relação à espessura obteve-se um revestimento homogêneo de 325,2 $\mu \mathrm{m}$ ao longo do seu comprimento, isso é devido ao uso de um braço robótico em sua aplicação.

$\mathrm{O}$ tratamento com laser de $\mathrm{CO}_{2}$ formou revestimentos com menos defeitos, menos porosidades pode ser observado o desaparecimento das partículas não fundidas no processo anterior comparado ao processo via HVOF.

No ensaio de microdureza, as amostras revestidas com WC sem irradiação a laser tiveram um valor próximo de $1600 \mathrm{HV}$. A amostra revestida com WC e irradiada com laser de $\mathrm{CO}_{2}$, apresentou um valor em torno de $1000 \mathrm{HV}$ no revestimento.

No ensaio de desgaste, as amostras apresentaram uma perda de volume em relação a amostra de aço 1020 de: 11,8 vezes menor em relação a amostra revestida com Carbeto de Tungstênio e irradiada com Laser de $\mathrm{CO}_{2}$ e 43,17 vezes menor em relação a amostra revestida com Carbeto de Tungstênio. A amostra apenas revestida com Carbeto de Tungstênio apresentou uma perda de volume de 3,65 vezes menor que a amostra revestida com WC irradiada com feixe de laser, devido a sua elevada microdureza.

A medida da rugosidade Ra, conforme os resultados da Tabela 3, destacou que a amostra apenas revestida com WC apresentou uma menor rugosidade média em relação à amostra irradiada com o feixe de laser. Ambas podem receber um processo de polimento, se necessário, para apresentar uma menor rugosidade.

\section{BIBLIOGRAFIA}

[1] PANZIERA, R. C., "Aplicação do revestimento de WC depositado por aspersão térmica pelo método HVOF para diminuir a abrasão do grão de arroz em máquinas agrícolas”, trabalho de conclusão de curso, Universidade Federal do Pampa - UNIPAMPA, Alegrete, RS, Brasil, 2016. 
[2] NUNES, R. A. X., COSTA, V. C., CALADO, V. M. D. A., et al., "Wear, friction, and microhardness of a thermal sprayed PET: poly (ethylene terephthalate) coating", Materials Research, v. 12, n.2, pp. 121-125, 2009.

[3] DAVIS, J. “Handbook of Thermal Spray Technology, ASM International Materials Park”, OH, 2004.

[4] LIMA, C., TREVISAN, R., “Aspersão Térmica: fundamentos e aplicações”, 2a Edição, São Paulo, Artliber Editora, 2007.

[5] CASTRO, R. D. M., CAVALER, L. C. D. C., KEJELIN, N. Z., et al., "Revestimento WC depositado por aspersão térmica (HVOF) como alternativa ao cromo duro eletrodepositado aplicados em equipamentos hidráulicos”, Revista Iberoamericana de Ingeniería Mecánica, v. 19, n. 2, pp. 27-42, 2015.

[6] GARCIA-ALONSO, D. SERRES, N., DEMIAN, C., et al., "Pre-/during-/post-laser processes to enhance the adhesion and mechanical properties of thermal-sprayed coatings with a reduced environmental impact”, Journal of Thermal Spray Technology, v. 20, n. 4, pp. 719-735, 2011.

[7] SIDHU, B. S., PURI, D., PRAKASH, S., "Characterisations of plasma sprayed and laser remelted NiCrAlY bond coats and Ni3Al coatings on boiler tube steels", Materials Science and Engineering: A, v. 368, n.1-2, pp. 149-158, 2004.

[8] TELEGINSKI, V., CHAGAS, D. C., COSTA DE OLIVEIRA, A. C., et al., "Yb:fiber laser surface texturing of stainless steel substrate, with MCrA1Y deposition and CO2 laser treatment", Surface and Coatings Technology, v. 260, pp. 251-259, 2014.

[9] CHIKARAKARA, E., PUNSET, M., PICAS, J. A., et al., "Characterisation of laser modified WC-CoCr coatings", AIP Conference Proceedings, v. 1353, n. 1, pp. 1087-1092, 2011.

[10] AFZAL, M., KHAN, A. N., BEN MAHMUD, T., et al., "Effect of laser melting on plasma sprayed WC12wt.\%Co coatings", Surface and Coatings Technology, v. 266, pp. 22-30, 2015.

[11] ZHANG, S. H., YOON, J. H., LI, M. X. , et al., "Influence of CO2 laser heat treatment on surface properties, electrochemical and tribological performance of HVOF sprayed WC-24\%Cr3C2-6\% Ni coating", $M a-$ terials Chemistry and Physics, v. 119, n. 3, pp. 458-464, 2010.

[12] GANEEV, R. A., "Low-power laser hardening of steels", Journal of materials processing technology, v. 121, n. 2-3, pp. 414-419, 2002.

[13] CONTIN, A., ALVES, K. A., CAMPOS, R. A., et al., "Diamond Films on Stainless Steel Substrates with an Interlayer Applied by Laser Cladding”, Materials Research, v. 20, n. 2, pp. 543-548, 2017.

[14] VAN ACKER, K., VANHOYWEGHEN, D., PERSOONS, R., et al., "Influence of tungsten carbide particle size and distribution on the wear resistance of laser clad WC/Ni coatings", Wear, v. 258, n. 1-4, pp. 194-202, 2005.

[15] BRANDOLT, C. D. S., ORTEGA VEGA, M. R., MENEZES, T. L., et al., "Corrosion behavior of nickel and cobalt coatings obtained by high- velocity oxy- fuel (HVOF) thermal spraying on API 5CT P110 steel", Materials and Corrosion, v. 67, n. 4, pp. 368-377, 2016.

[16] MATEOS, J., CUETOS, J. M., FERNÁNDEZ, E., et al., “Tribological behaviour of plasma sprayed WC coatings with and without laser remelting”, Wear, v. 239, n. 2, pp. 274-281, 2000.

\section{ORCID}

Renato Camponogara Panziera Ana Claudia Costa de Oliveira https://orcid.org/0000-0002-2043-8849

https://orcid.org/0000-0002-2437-8425 